\title{
CRITICAL SETS IN PARAMETRIC OPTIMIZATION
}

\author{
H.Th. JONGEN, P. JONKER and F. TWILT \\ Twente University of Technology, Department of Applied Mathematics, P.O. Box 217, \\ 7500 AE Enschede, The Netherlands
}

\begin{abstract}
We deal with one-parameter families of optimization problems in finite dimensions. The constraints are both of equality and inequality type. The concept of a 'generalized critical point' (g.c. point) is introduced. In particular, every local minimum, Kuhn-Tucker point, and point of Fritz John type is a g.c. point. Under fairly weak (even generic) conditions we study the set $\Sigma$ consisting of all g.c. points. Due to the parameter, the set $\Sigma$ is pieced together from one-dimensional manifolds. The points of $\Sigma$ can be divided into five (characteristic) types. The subset of 'nondegenerate critical points' (first type) is open and dense in $\Sigma$ (nondegenerate means: strict complementarity, nondegeneracy of the corresponding quadratic form and linear independence of the gradients of binding constraints). A nondegenerate critical point is completely characterized by means of four indices. The change of these indices along $\Sigma$ is presented. Finally, the Kuhn-Tucker subset of $\Sigma$ is studied in more detail, in particular in connection with the (failure of the) Mangasarian-Fromowitz constraint qualification.
\end{abstract}

Key words: Generalized Critical Point, Critical Point, Linear Index, Quadratic Index, KuhnTucker Set, Mangasarian-Fromowitz Constraint Qualification, Parametric Optimization.

\section{Introduction}

Let $C^{k}\left(\mathbb{R}^{n}, \mathbb{R}\right), k \geqslant 1$, denote the space of real valued, $k$-times continuously differentiable functions defined on the $n$-dimensional Euclidean space $\mathbb{R}^{n}, n \geqslant 1$. Given finite index sets $I, J$, a differentiable optimization problem $\mathscr{P}$ has the following standard formulation:

$$
\mathscr{P}: \quad \text { Minimize } f \text { on } M \text {, }
$$

where

$$
M=\left\{x \in \mathbb{R}^{n} \mid h_{i}(x)=0, g_{j}(x) \geqslant 0, i \in I, j \in J\right\},
$$

and $f, h_{i}, g_{j} \in C^{2}\left(\mathbb{R}^{n}, \mathbb{R}\right), i \in I, j \in J$.

The function $f$ is the objective function, $h_{i}\left(g_{j}\right)$ are the (in)equality constraints and $M$ is the feasible set.

Our paper is a study of one-parameter families of optimization problems of the type (1). The motivation for this is manifold and we start with a short exposition of our incentives.

Currently, there is a growing interest in the subject of sensitivity and stability analysis of mathematical programming problems. Important contributions are contained in $[3,4]$. One-parameter families play a special role in this area. In fact, within a path-connected set of perturbation parameters two problems can be joined 
by means of a one-parameter family. Moreover, a detailed description of the generic behaviour of problem $\mathscr{P}$ depending on one-parameter can be given, as will be shown in this paper. However, such a description becomes extremely difficult if more parameters are involved. The latter fact is related with complicated phenomena in the theory of singularities of higher 'singularity-codimension'; see [2] and, in particular, also [15]. Of course, there are many problems where the number of parameters is essentially greater than one, such as, for example problems of vectoroptimization. But also in this case one-parameter families play a role as being one-dimensional sections of the total parametric problem; see [5]. If we look at one-parameter families as deformations of one problem into another one, we have an intimate relation with continuation, resp. homotopy methods; see [1] for an extensive survey. Finally, interpreting the parameter as time, we obtain an insight into the dynamic behaviour of problem $\mathscr{P}$.

In [9] we studied the generic behaviour of the feasible set depending on one parameter, from both a local and global point of view. Now we proceed by investigating the structure of the set of 'critical' points (for the special case of equality constraints only, see [11]). Our concept of a 'critical' point will be a quite general one. But it turns out that it is very suitable when studying parametric problems.

For $\phi \in C^{1}\left(\mathbb{R}^{n}, \mathbb{R}\right)$ let $D \phi(x)$ denote the row vector of the first partial derivatives at $x$ and let $J_{0}(x)$ denote the index set of active (=binding) inequality constraints:

$$
J_{0}(x)=\left\{j \in J \mid g_{j}(x)=0\right\} .
$$

Definition 1.1. A point $\bar{x} \in \mathbb{R}^{n}$ is called a generalized critical point (g.c. point) for $\mathscr{P}$ (or for $\left.f\right|_{M}$ ), if $\bar{x}$ belongs to $M$ and, moreover, if the set of vectors

$$
\left.\left\{D f, D h_{i}, D g_{j}, i \in I, j \in J_{0}(\bar{x})\right\}\right|_{x=\bar{x}}
$$

is linearly dependent.

If $\bar{x}$ is a g.c. point for $\mathscr{P}$, then obviously there exist real numbers $\lambda, \lambda_{i}, \mu_{j}, i \in I$, $j \in J_{0}(\bar{x})$, not all vanishing, such that

$$
\lambda D f=\sum_{i \in I} \lambda_{i} D h_{i}+\left.\sum_{j \in J_{0}(\bar{x})} \mu_{j} D g_{j}\right|_{x=\bar{x}}
$$

In case that $\left(\lambda, \lambda_{i}, \mu_{j}\right)$ in (5) can be chosen such that $\lambda>0, \mu_{j} \geqslant 0, j \in J_{0}(\bar{x})$ resp. $\lambda \geqslant 0, \mu_{j} \geqslant 0, j \in J_{0}(\bar{x})$, the point $\bar{x}$ is usually called a Kuhn-Tucker point, resp. a point of Fritz John type. In particular, a local minimum $\bar{x}$ for $\mathscr{P}$ is always a point of Fritz John type (cf. [6]). However, it need not be a Kuhn-Tucker point, unless some constraint qualification is satisfied. The simplest constraint qualification is linear independence of the set $\left.\left\{D h_{i}, D g_{j}, i \in I, j \in J_{0}(\bar{x})\right\}\right|_{x=\bar{x}}$. In the latter case, the number $\lambda$ in (5) must be unequal to zero and, moreover, the set $M$ is locally $C^{2}$-diffeomorphic to $\mathbb{R}^{p} \times \mathbb{H}^{q}$, where $\mathbb{H}^{q}$ is the nonnegative orthant in $\mathbb{R}^{q}$ and $p=n-|I|-\left|J_{0}(\bar{x})\right|, q=\left|J_{0}(\bar{x})\right|$ (cf. [10]). This gives rise to the following definition. 
Definition 1.2. The set $M$ in (2) is called regular at $\bar{x}, \bar{x} \in M$, if $\left\{D h_{i}, D g_{j}, i \in I\right.$, $\left.j \in J_{0}(\bar{x})\right\}\left.\right|_{x=\bar{x}}$ is a linearly independent set. Moreover, a generalized critical point $\bar{x}$ is called a critical point (for $\mathscr{P}$ or $\left.f\right|_{M}$ ) if $M$ is regular at $\bar{x}$.

In order to give an outline of the paper we need one more definition ("nondegenerate' critical point). For $\phi \in C^{2}\left(\mathbb{R}^{n}, \mathbb{R}\right)$ we denote by $D^{2} \phi(x)$ the (symmetric) matrix of the second order partial derivatives at $x$ (i.e. the Hessian). If $A$ is a symmetric $n \times n$ matrix and $L$ a linear subspace of $\mathbb{R}^{n}$, then by $\left.A\right|_{L}$ we mean some matrix of the family $\mathscr{V}, \mathscr{V}=\left\{V^{\mathrm{T}} A V \mid V\right.$ is a matrix with $n$ rows, whose columns form a basis for $L$ \}. In view of Sylvester's theorem (cf. [13]), the number of negative, resp. zero, positive eigenvalues of $V^{\mathrm{T}} A V$ does not depend on the incidental choice of $V$. Therefore, the number of negative, resp. zero, positive eigenvalues of $\left.A\right|_{L}$ is defined to be the corresponding number of $V^{\mathrm{T}} A V$, where $V^{\mathrm{T}} A V \in \mathscr{V}$. Furthermore, $\left.A\right|_{L}$ is said to be nonsingular if $V^{\mathrm{T}} A V$ is nonsingular, where $V^{\mathrm{T}} A V \in \mathscr{V}$.

For an $r \times q$ matrix $B$, the set $\operatorname{Ker} B$ will be

$$
\operatorname{Ker} B=\left\{\xi \in \mathbb{R}^{q} \mid B \xi=0\right\} \text {. }
$$

Definition 1.3. Let $M$ be regular at $\bar{x}$ and let $\bar{x}$ be a critical point for $\left.f\right|_{M}$, i.e. there exist (Lagrange parameters) $\bar{\lambda}_{i}, \bar{\mu}_{j}, i \in I, j \in J_{0}(\bar{x})$, such that

$$
D f=\sum_{i \in I} \bar{\lambda}_{i} D h_{i}+\left.\sum_{j \in J_{0}(\bar{x})} \bar{\mu}_{j} D g_{j}\right|_{x=\bar{x}} .
$$

The critical point $\bar{x}$ is called nondegenerate if the following two conditions hold:

$N D 1: \bar{\mu}_{j} \neq 0, j \in J_{0}(\bar{x})$,

$N D 2:\left.D^{2} L(\bar{x})\right|_{T}$ is nonsingular,

where ( $L=$ Lagrange function, $T=$ tangent space)

$$
\begin{aligned}
& L(x)=f(x)-\sum_{i \in I} \bar{\lambda}_{i} h_{i}(x)-\sum_{j \in J_{0}(\bar{x})} \bar{\mu}_{j} g_{j}(x), \\
& T=\bigcap_{i \in I} \operatorname{Ker} D h_{i}(\bar{x}) \cap \bigcap_{j \in J_{0}(\bar{x})} \operatorname{Ker} D g_{j}(\bar{x}) .
\end{aligned}
$$

The linear index LI, resp. linear coindex LCI, is defined to be the number of $\bar{\mu}_{j}$ in (7) which are negative, resp. positive. The quadratic index QI, resp. quadratic coindex QCI, is defined to be the number of negative, resp. positive eigenvalues of $\left.D^{2} L(\bar{x})\right|_{T}$, with $L, T$ as in (8), (9).

The numbers LI, LCI, QI, QCI at a nondegenerate critical point $\bar{x}$ completely characterize the local behaviour of $\left.f\right|_{M}$ and in this sense they are intrinsic (cf. [10] for a detailed exposition). In particular, if $\mathrm{LI}=\mathrm{QI}=0$, resp. $\mathrm{LCI}=\mathrm{QCI}=0$, then $\bar{x}$ is a local minimum, resp. local maximum for $\left.f\right|_{M}$. In all other cases, $\bar{x}$ is a certain kind of saddle-point. If $\mathrm{LI}=0$, then $\bar{x}$ is a Kuhn-Tucker point and in that case the quadratic index is the appropriate generalization of the so called Morse-index (cf. $[10,14])$. 
In the sequel we consider the following setting of a one-parameter family of problems, where a vector $z \in \mathbb{R}^{n+1}$ is always partitioned as $z=(x, t), x \in \mathbb{R}^{n}, t \in \mathbb{R}$ $(t=$ parameter):

$$
\mathscr{P}(t): \quad \text { Minimize } f(\cdot, t) \text { on } M(t) \quad(t \in \mathbb{R})
$$

where

$$
\begin{aligned}
& M(t)=\left\{x \in \mathbb{R}^{n} \mid h_{i}(x, t)=0, g_{j}(x, t) \geqslant 0, i \in I, j \in J\right\}, \\
& I=\{1, \ldots, m\}, \quad m<n, \quad J=\{1, \ldots, s\}, \\
& f, h_{i}, g_{j} \in C^{3}\left(\mathbb{R}^{n+1}, \mathbb{R}\right), \quad i \in I, j \in J .
\end{aligned}
$$

A point $\bar{z} \in \mathbb{R}^{n+1}$ is called a (generalized, nondegenerate) critical point for $\mathscr{P}(\cdot)$ if $\bar{x}$ is a (generalized, nondegenerate) critical point for $\mathscr{P}(\bar{t})$, where $\bar{z}=(\bar{x}, \bar{t})$. The set of all generalized critical points will be denoted by $\Sigma$, so

$$
\Sigma=\left\{z \in \mathbb{R}^{n+1} \mid x \text { is g.c. point for } \mathscr{P}(t) \text {, where } z=(x, t)\right\} .
$$

Roughly speaking, for most problems $\mathscr{P}(\cdot)$ it turns out that most points of $\Sigma$ are nondegenerate critical points (points of Type 1 in the subsequent terminology). This observation is in a certain sense connected with an interesting study in [16]. However, one cannot expect that all points of $\Sigma$ are nondegenerate critical points. In fact, for some parameter values $t$ it might happen that at some points $x$ the regularity of the set $M(t)$ is not satisfied (if the number of active constraints is less than $(n+1)$ we are dealing with a point of Type 4; if this number equals $(n+1)$ we will have a point of Type 5). So, this is one 'degeneracy' phenomenon. Other degeneracies can take place at critical points (so $M(t)$ is regular) where one of the conditions ND1, ND2 does not hold. Note, if ND1 fails to hold, then the 'strict complementarity' is not satisfied (Type 2). Further, if ND2 is not satisfied, then one of the eigenvalues of $\left.D^{2} L(\bar{x})\right|_{T}$ vanishes (Type 3). We emphasize that the degeneracies (Type 2, 3, 4, 5) are stable, i.e. they remain present under ' $C^{3}$-perturbations' of the problemdefining functions $f, h_{i}, g_{j}$. The set $\Sigma$ will generically be one-dimensional. Walking along $\Sigma$ we will meet at certain (discrete) points a degeneracy (Type 2, 3, 4, 5). A basic question then arises: if we pass a point of Type 2, 3, 4, 5, what effect does this have on the local structure of the nondegenerate critical points before and after passing; i.e. how does the set of indices LI, LCI, QI, QCI change?

An important related study on Kuhn-Tucker points is done in [12], where it is assumed that the Mangasarian-Fromowitz constraint qualification holds (cf. Section 4). Finally we remark that 'Kuhn-Tucker' branches of the set $\Sigma$ might be connected by means of non-Kuhn-Tucker branches (see [8]). In a certain sense this is analogous to the complexification-effect of a one-parameter family of real analytic systems (see [1, Section 4]).

The paper is organized as follows. In Section 2 we state a genericity theorem which clarifies the concept 'most problems $\mathscr{P}(\cdot)$ '. In Section 3 we describe in five subsections the local behaviour of the set $\Sigma$ in a neighbourhood of each of the 
points of Type 1-5 (including the change of the indices LI, LCI, QI, QCI). Finally, in Section 4 we concentrate on the (closure of the) Kuhn-Tucker subset and discuss those situations where the Mangasarian-Fromowitz constraint qualification is not satisfied.

\section{The genericity theorem}

In this section we adopt the notation of Section 1 . The space $C^{3}\left(\mathbb{R}^{n+1}, \mathbb{R}\right)$ will be endowed with the strong (or Whitney-) $C_{S}^{3}$-topology (cf. [7]), the $C_{S}^{3}$-topology of the product of a finite number of copies of $C^{3}\left(\mathbb{R}^{n+1}, \mathbb{R}\right)$ being the induced product topology. A typical $C_{S}^{3}$ base-neighbourhood $\mathcal{N}_{\varepsilon}$ of the zero function in $C^{3}\left(\mathbb{R}^{n+1}, \mathbb{R}\right)$ is induced by means of a continuous positive function $\varepsilon: \mathbb{R}^{n+1} \rightarrow \mathbb{R}$ as follows:

$$
\begin{aligned}
\mathcal{N}_{\varepsilon}=\left\{\phi \in C^{3}\left(\mathbb{R}^{n+1}, \mathbb{R}\right)|| \phi(z) \mid\right. & +\Sigma\left|\frac{\partial}{\partial z_{i}} \phi(z)\right|+\Sigma\left|\frac{\partial^{2}}{\partial z_{i} \partial z_{j}} \phi(z)\right| \\
& \left.+\Sigma\left|\frac{\partial^{3}}{\partial z_{i} \partial z_{j} \partial z_{k}} \phi(z)\right|<\varepsilon(z) \text { for all } z \in \mathbb{R}^{n+1}\right\} .
\end{aligned}
$$

A typical $C_{S}^{3}$ base-neighbourhood of $f \in C^{3}\left(\mathbb{R}^{n+1}, \mathbb{R}\right)$ will be the set $f+\mathcal{N}_{\varepsilon}$.

In Section 1 we intuitively introduced points of Type 1-5. These types will be made precise in the next section. Taking this for granted at this stage we can proceed with the statement of the genericity theorem.

Definition 2.1. Let the subset $\mathscr{F}$ of $C^{3}\left(\mathbb{R}^{n+1}, \mathbb{R}\right)^{1+m+s}$ be defined as follows: $\left(f, h_{1}, \ldots, h_{m}, g_{1}, \ldots, g_{s}\right) \in \mathscr{F}$ if each point of $\Sigma$ (cf. (13)) is one of the Types 1,2 , $3,4,5$.

Theorem 2.1 (Genericity). The set $\mathscr{F}$ is $C_{S}^{3}$ open and dense in $C^{3}\left(\mathbb{R}^{n+1}, \mathbb{R}\right)^{1+m+s}$.

The dense-part of the proof of Theorem 2.1 is tedious and based on transversal approximation. A sketch of the proof in case $J=\emptyset$ (i.e. without inequality constraints) is given in [11] and that proof can be extended without severe difficulties to a proof of the present situation. The open-part of the proof of Theorem 2.1 can be accomplished by means of continuity arguments (note that $C^{3}$-differentiability is needed in view of points of Type 3). We define:

$$
\Sigma^{i}=\{z \in \Sigma \mid z \text { is of Type } i\}, \cdot i=1, \ldots, 5 \text {. }
$$

Theorem 2.2. Let $\left(f, h_{1}, \ldots, h_{m}, g_{1}, \ldots, g_{s}\right)$ belong to $\mathscr{F}$. Then we have: $\Sigma^{1}$ is open and dense in $\Sigma$ and, for $i=2,3,4,5$, the set $\Sigma^{i}$ is a discrete point set.

The proof of Theorem 2.2 is a straightforward consequence of the local structure of the set $\Sigma$ in a neighbourhood of the points of Type 1-5, as will be explained in the next section. 


\section{Analysis of the five types}

In this section we will make an analysis on the points of Type 1-5 which we intuitively introduced in Section 1. To this aim we divide this section into five subsections, each of them being dedicated to one single type. Every subsection is organized as follows. Firstly, we state a number of conditions which are necessary to describe the type under consideration, and we introduce so-called characteristic numbers which determine the essence of the type. Based on this information we proceed with a local description of the set $\Sigma$ and the index-relations involved.

The notation of the foregoing sections will be adopted. In particular, a vector $z \in \mathbb{R}^{n+1}$ will always be partitioned as $z=(x, t), x \in \mathbb{R}^{n}, t \in \mathbb{R}$, and $J_{0}(z)$ denotes the index set of active (=binding) inequality constraints. For $\phi \in C^{k}\left(\mathbb{R}^{n+1}, \mathbb{R}\right), D_{x} \phi$ stands for the row vector of first partial derivatives with respect to $x ; D_{t} \phi, D_{x}^{2} \phi$ are to be interpreted analogously.

\subsection{Points of type 1}

A generalized critical point $\bar{z}=(\bar{x}, \bar{t})$ is of Type 1 if $\bar{z}$ is a nondegenerate critical point, i.e. $\bar{x}$ is a nondegenerate critical point for $\mathscr{P}(\bar{t})$.

Characteristic numbers: LI, LCI, QI, QCI.

Let $\bar{z}=(\bar{x}, \bar{t})$ be a point of Type 1 . Then, in an $\left(\mathbb{R}^{n+1}-\right)$ neighbourhood of $\bar{z}$ we can parametrize the set $\Sigma$ by means of the parameter $t$, whereas the indices LI, LCI, QI, QCI remain (locally) constant. To see this, we use the implicit function theorem. Without loss of generality we may assume, in case $J_{0}(\bar{z}) \neq \emptyset$, that $J_{0}(\bar{z})=$ $\{1, \ldots, p\}$. Let $\lambda$, resp. $\mu$, be an $m$, resp. $p$ vector, with components $\lambda_{i}$, resp. $\mu_{j}$. Consider the map $\mathscr{T}: \mathbb{R}^{n+m+p+1} \rightarrow \mathbb{R}^{n+m+p}$,

$$
\mathscr{T}:\left(\begin{array}{c}
x \\
\lambda \\
\mu \\
t
\end{array}\right) \mapsto\left(\begin{array}{c}
D_{x}^{\mathrm{T}} f(x, t)-\sum_{i=1}^{m} \lambda_{i} D_{x}^{\mathrm{T}} h_{i}(x, t)-\sum_{j=1}^{p} \mu_{j} D_{x}^{\mathrm{T}} g_{j}(x, t) \\
h_{i}(x, t), i=1, \ldots, m \\
g_{j}(x, t), j=1, \ldots, p
\end{array}\right)
$$

The map $\mathscr{T}$ in (15) is of class $C^{2}$ since $f, h_{i}, g_{j}$ are of class $C^{3}$. Let $\left(\bar{\lambda}_{i}\right),\left(\bar{\mu}_{j}\right)$ be the Lagrange parameters at $\bar{x}$ as a critical point for $\mathscr{P}(\bar{t})$ (cf. (7)). Then $\mathscr{T}$ vanishes at the point $(\bar{x}, \bar{\lambda}, \bar{\mu}, \bar{t})$. The partial derivative of $\mathscr{T}$ at $(\bar{x}, \bar{\lambda}, \bar{\mu}, \bar{t})$ with respect to $(x, \lambda, \mu)$ has the following typical blockstructure:

$$
\left(\begin{array}{c|c}
D_{x}^{2} L(\bar{z}) & -B \\
\hline B^{T} & 0
\end{array}\right)
$$

where

$$
L(z)=f(z)-\sum_{i=1}^{m} \bar{\lambda}_{i} h_{i}(z)-\sum_{j=1}^{p} \bar{\mu}_{j} g_{j}(z)
$$

and $B=\left.\left(D_{x}^{\mathrm{T}} h_{1}|\cdots| D_{x}^{\mathrm{T}} g_{p}\right)\right|_{z=\bar{z}}$. 
Note that the matrix $B$ in (16) has rank $(m+p)$. Then, from condition ND2 (cf. Definition 1.3) it follows that the matrix in (16) is nonsingular (see [10]). Now we can apply the implicit function theorem and obtain $C^{2}$-mappings $x(t), \lambda(t), \mu(t)$ (in an open neighbourhood of $\bar{t}$ ) such that $\mathscr{T}(x(t), \lambda(t), \mu(t), t) \equiv 0$. Note that all components of $\bar{\mu}$ are unequal to zero (condition ND1) and that $\mu(\bar{t})=\bar{\mu}$. Consequently, for $t$ sufficiently close to $\bar{t}$ we see that no component of $\mu(t)$ vanishes. In an open neighbourhood of $\bar{z}$ we can parametrize the set $\Sigma$ by means of a unique $C^{2}$-map $t \mapsto(x(t), t)$. Hence, $\Sigma^{1}$ is a one-dimensional $C^{2}$-manifold and the indices LI, LCI, QI, QCI are constant on every connected component of $\Sigma^{1}$.

\subsection{Points of Type 2}

A generalized critical point $\bar{z}=(\bar{x}, \bar{t})$ is of Type 2 if the following conditions A1-A6 hold:

A1. $\bar{x}$ is a critical point for $\mathscr{P}(\bar{t})$.

A2. $J_{0}(\bar{z}) \neq \emptyset$.

After renumbering we may assume that $J_{0}(\bar{z})=\{1, \ldots, p\}, p \geqslant 1$. Then, we have (cf. A1 and (7))

$$
D_{x} f=\sum_{i=1}^{m} \bar{\lambda}_{i} D_{x} h_{i}+\left.\sum_{j=1}^{p} \bar{\mu}_{j} D_{x} g_{j}\right|_{z=\bar{z}} .
$$

A3. In (18) exactly one of the Lagrange parameters $\bar{\mu}_{j}$ vanishes.

After renumbering we may assume that $\bar{\mu}_{p}=0$ and $\bar{\mu}_{j} \neq 0, j=1, \ldots, p-1$. Let the Lagrange function $L$ be defined as in (17) and put

$$
\begin{aligned}
& T=\bigcap_{i \in I} \operatorname{Ker} D_{x} h_{i}(\bar{z}) \cap \bigcap_{j \in J_{0}(\bar{z})} \operatorname{Ker} D_{x} g_{j}(\bar{z}), \\
& \tilde{T}=\bigcap_{i \in I} \operatorname{Ker} D_{x} h_{i}(\bar{z}) \cap \bigcap_{j \in J_{0}(\bar{z}) \backslash\{p\}} \operatorname{Ker} D_{x} g_{j}(\bar{z}) .
\end{aligned}
$$

A4. $\left.D_{x}^{2} L(\bar{z})\right|_{T}$ is nonsingular.

A5. $\left.D_{x}^{2} L(\bar{z})\right|_{\tilde{T}}$ is nonsingular.

Let $B$ be an $n \times r$-matrix of rank $r$. By $B^{\dagger}$ we denote the matrix $\left(B^{\mathrm{T}} B\right)^{-1} B^{\mathrm{T}}$. In fact, $B^{\dagger}$ is the Moore-Penrose inverse of $B$.

Let $W$ be a matrix with $n$ rows, whose columns form a basis for the linear space $\tilde{T}$. Put $\Phi=\left(h_{1}, \ldots, h_{m}, g_{1}, \ldots, g_{p-1}\right)^{\mathrm{T}}$ and define the $n \times 1$-vectors:

$$
\begin{aligned}
& \alpha=-\left(\left(D_{x}^{\mathrm{T}} \Phi\right)^{\dagger}\right)^{\mathrm{T}} \cdot D_{t} \Phi, \\
& \beta=-W\left(W^{\mathrm{T}} \cdot D_{x}^{2} L \cdot W\right)^{-1} W^{\mathrm{T}}\left\{D_{x}^{2} L \cdot \alpha+D_{t} D_{x}^{\mathrm{T}} L\right\} .
\end{aligned}
$$

In (21), (22), $D_{t}$ stands for $\partial / \partial t$ and all partial derivatives are evaluated at $\bar{z}$. Next, we put

$$
\gamma=D_{x} g_{p}(\bar{z})(\alpha+\beta)+D_{t} g_{p}(\bar{z})
$$

Note that $\beta$ (and thus $\gamma$ as well) is independent of the choice of the matrix $W$. A6. $\gamma \neq 0$. 
Let $\delta_{1}$, resp. $\delta_{2}$ denote the number of negative eigenvalues of $\left.D_{x}^{2} L(\bar{z})\right|_{\tilde{T}}$, resp. $\left.D_{x}^{2} L(\bar{z})\right|_{T}$, and put $\delta=\delta_{1}-\delta_{2}$.

Characteristic numbers: $\operatorname{sign}(\gamma), \delta$.

In the special case that $I=\emptyset$ and $p=1$, we have $\tilde{T}=\mathbb{R}^{n}$ (cf. A5) and we just delete all entries of $\Phi$ in (21).

We proceed with an analysis of the set $\Sigma$ in a neighbourhood of $\bar{z}$.

The simplest example to have in mind is the following (one-dimensional): $f(x, t)$ is one of the four functions $\pm(x \pm t)^{2}$, there is only one constraint, namely the constant inequality constraint $x \geqslant 0$, and the point $\bar{z}$ is the origin in $\mathbb{R}^{2}$. Note that, as $t$ passes zero, an unconstrained critical point is moving into the feasible set or out of the feasible set. (A similar higher dimensional example is obtained by choosing $f(x, t)$ from the functions $\pm\left(x_{n} \pm t\right)^{2}+\sum_{j=1}^{n-1} \pm x_{j}^{2}$ and taking only one constraint, namely $x_{n} \geqslant 0$ into account).

It will turn out that, locally, $\Sigma$ is pieced together from two curves which intersect at $\bar{z}$. In fact, since $\bar{\mu}_{p}$ vanishes, it follows that $\bar{x}$ is a critical point both for problem $\mathscr{P}(\bar{t})$ as well as for the problem $\tilde{\mathscr{P}}(\bar{t})$, where $\tilde{\mathscr{P}}(\bar{t})$ differs from $\mathscr{P}(\bar{t})$ only in the fact that the inequality constraint $g_{p}$ is deleted. The Lagrange parameters $\bar{\mu}_{1}, \ldots, \bar{\mu}_{p-1}$ are unequal to zero.

Together with condition A5 it follows that $\bar{x}$ is a nondegenerate critical point for $\tilde{\mathscr{P}}(\bar{t})$. Now we can apply the result of Section 3.1 to problem $\tilde{\mathscr{P}}(\cdot)$. Let $\tilde{\Sigma}$ denote the set of g.c. points for problem $\tilde{\mathscr{P}}(\cdot)$. Then, in a neighbourhood of $\bar{z}$ the set $\tilde{\Sigma}$ is a one-dimensional manifold, parametrized by means of a unique $C^{2}$-map $t \mapsto$ $(\tilde{x}(t), t)$. The latter curve belongs to $\Sigma$ as far as $\psi(t)$ is nonnegative, where $\psi(t):=$ $g_{p}(\tilde{x}(t), t)$, and it traverses the zero set ' $g_{p}=0$ ' transversally iff $(\mathrm{d} \psi / \mathrm{d} t)(\bar{t}) \neq 0$. A few calculations show that $(\mathrm{d} \tilde{x} / \mathrm{d} t)(\bar{t})=\alpha+\beta($ cf. (21), (22)). Hence $(\mathrm{d} \psi / \mathrm{d} t)(\bar{t})=\gamma$, with $\gamma$ as in (23). So, if we walk along $\tilde{\Sigma}$ as $t$ increases, then at $t=\bar{t}$ we leave (enter) the feasible set $M(t)$ (cf. (11)) according to $\operatorname{sign}(\gamma)=-1(+1)$.

Next, we consider a problem $\hat{\mathscr{P}}(\bar{t})$ which differs from $\mathscr{P}(\bar{t})$ only in the fact that $g_{p}$ is treated as an equality constraint. Of course, $\bar{x}$ is also a critical point for $\hat{\mathscr{P}}(\bar{t})$ and, moreover, $\bar{x}$ is a nondegenerate critical point for $\hat{\mathscr{P}}(\bar{t})$. In fact, the nondegeneracy condition ND1 (cf. Definition 1.3) is satisfied, since the vanishing Lagrange parameter $\bar{\mu}_{p}$ does not anymore correspond to an inequality constraint (by the very definition of $\hat{\mathscr{P}}(\bar{t})$ ). Condition ND2 holds in view of A4. Let $\hat{\Sigma}$ be the set of g.c. points for problem $\hat{\mathscr{P}}(\cdot)$. Again we can apply the result of Section 3.1 and hence, in a neighbourhood of $\bar{z}$ the set $\hat{\Sigma}$ is a one-dimensional manifold, parametrized by means of a unique $C^{2}$-map $t \mapsto(\hat{x}(t), t)$. (Note that $\left.g_{p}(\hat{x}(t), t) \equiv 0\right)$. Since the curve $\tilde{\Sigma}$ traverses the zero set ' $g_{p}=0$ ' at $\bar{z}$ transversally, it follows that $\tilde{\Sigma}$ and $\hat{\Sigma}$ intersect at $\bar{z}$ under a nonvanishing angle. Obviously, in a neighbourhood of $\bar{z}$, the set $\Sigma$ consists of $\hat{\Sigma}$ and that part of $\tilde{\Sigma}$ on which $g_{p}$ is nonnegative. Altogether, the set $\Sigma$ has a local structure as depicted in Fig. 1c,d, and note that $\Sigma \backslash\{\bar{z}\}$ consists of nondegenerate critical points.

We proceed with the calculation of the index-relations between the branches of nondegenerate critical points in $\Sigma \backslash\{\bar{z}\}$. Let us consider the (above defined) curve 


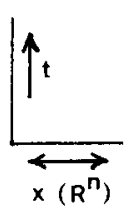

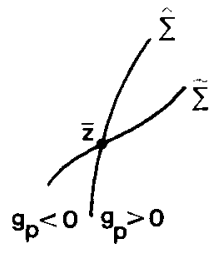

a

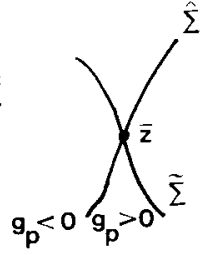

b

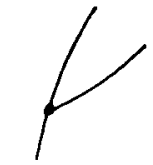

$Y>0$

c

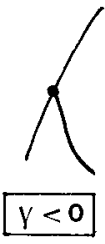

d

Fig. 1.

$t \mapsto(\hat{x}(t), t)$ once more, i.e. we treat $g_{p}$ as an equality constraint. For each $t$ we can calculate the Lagrange parameter corresponding to $g_{p}$, say $\mu_{p}(t)$, at the point $\hat{x}(t)$, viewed at as a critical point for $\hat{\mathscr{P}}(t)$. Since all data are of class $C^{3}, \mu(\cdot)$ will be of class $C^{2}$. Now, if we look at $g_{p}$ as an inequality constraint, then, except for $t=\bar{t}$, the point $\hat{x}(t)$ is a nondegenerate critical point for $\mathscr{P}(t)$. In fact, the only difference between two critical points $\hat{x}\left(t_{1}\right), \hat{x}\left(t_{2}\right)$ for $\mathscr{P}(t)$, where $t_{1}<\bar{t}<t_{2}$, lies in the fact that one Lagrange parameter $\left(\mu_{p}(t)\right)$ belonging to an inequality constraint $\left(g_{p}\right)$ changes sign. In particular, we will show the more general relation:

$$
\operatorname{sign}(\gamma) \cdot \operatorname{sign}\left(\frac{\mathrm{d} \mu_{p}}{\mathrm{~d} t}(\bar{t})\right)=+1 \quad(\text { resp. }-1) \quad \text { iff } \delta=1 \quad(\text { resp. } \delta=0),
$$

where $\operatorname{sign}(\gamma)$ and $\delta$ are the characteristic numbers.

Taking (24) for granted at this stage and noting that the dimensions of $\tilde{T}$ and $T$ (cf. (19), (20)) differ by one, the index-relations are easily derived. In Fig. 2 we made a picture of all four possibilities; the 4-vectors stand for (LI, LCI, QI, QCI). Note that $\mathrm{LI}+\mathrm{LCI}+\mathrm{QI}+\mathrm{QCI}=n-m$, whereas $\mathrm{LI}+\mathrm{LCI}$ equals $p$ or $p-1$.

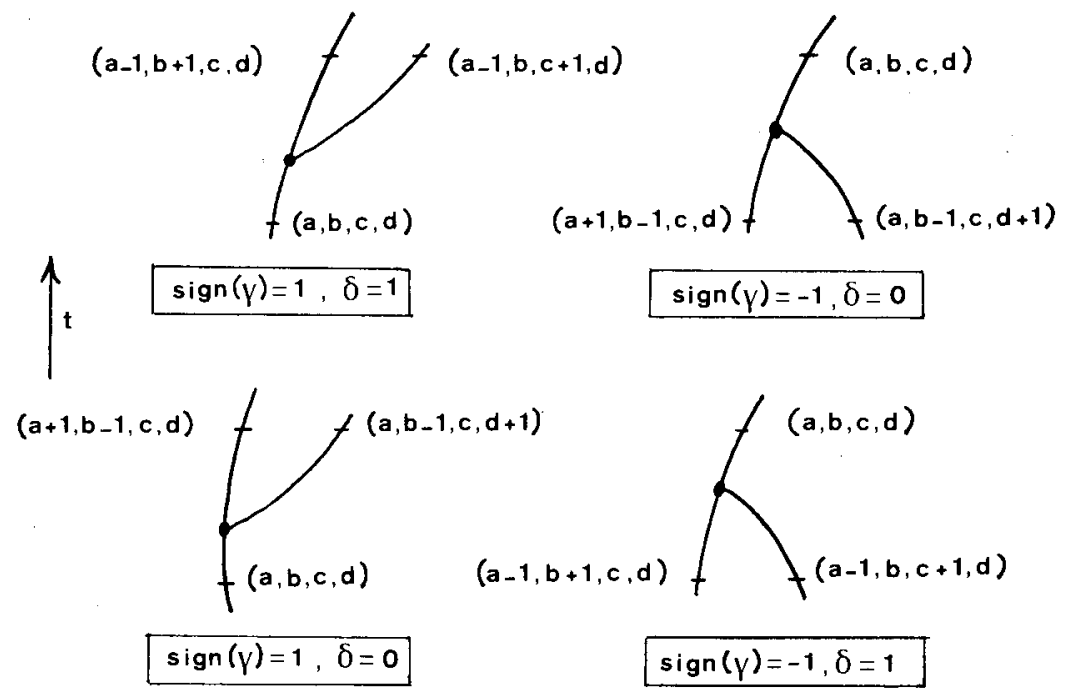

Fig. 2. 
In order to derive (24) we start with the following observation: the inequality constraints $g_{1}, \ldots, g_{p-1}$ remain active (locally) along $\tilde{\Sigma}$ and $\hat{\Sigma}$. Consequently, the only inequality constraint function that really counts is the function $g_{p}$. So, we can simplify the verification of (24) in local coordinates in a neighborhood of the origin in $\mathbb{R}^{q+1}$, where $q=\operatorname{dim} \tilde{T}=n-m-p+1$. In these new coordinates, say $(y, u)=$ $\Psi(x, t)$, ' $u$-hyperplanes' correspond to ' $t$-hyperplanes' and the orientation of $t$ is preserved.

In fact, choose vectors $\xi_{i} \in \mathbb{R}^{n}, i=1, \ldots, q-1$, which form together with the set $\left\{D_{x}^{\mathrm{T}} h_{i}(\bar{z}), D_{x}^{\mathrm{T}} g_{j}(\bar{z}), i=1, \ldots, m, j=1, \ldots, p\right\}$ a basis for $\mathbb{R}^{n}$, and consider the local $C^{3}$-coordinate transformation in $\mathbb{R}^{n+1},\left(y_{1}, \ldots, y_{n}, u\right)=\Psi\left(x_{1}, \ldots, x_{n}, t\right)$, defined by

$$
\begin{aligned}
& y_{i}=\xi_{i}^{\mathrm{T}}(x-\bar{x}), \quad i=1, \ldots, q-1, \\
& y_{q}=g_{p}(x, t), \\
& y_{q+j}=g_{j}(x, t), \quad j=1, \ldots, p-1, \\
& y_{q+p+i-1}=h_{i}(x, t), \quad i=1, \ldots, m, \\
& u=t-\bar{t} .
\end{aligned}
$$

Note that $(\bar{x}, \bar{t})$ is sent to the origin, and that, for $t$ close to $\bar{t}$ and $x$ close to $\bar{x}$, the intersection of the feasible set $M(t)$ with the zero set ( $t$ fixed): $g_{1}=\cdots=g_{p-1}=h_{1}=$ $\cdots=h_{m}=0$, is transformed to the following set of dimension $q$ (constant with respect to $u$ ):

$$
\left\{\left(y_{1}, \ldots, y_{q}\right) \in \mathbb{R}^{q} \mid y_{q} \geqslant 0\right\} .
$$

Let us denote the transformed function $f(x, t)$ by $g\left(y_{1}, \ldots, y_{q}, u\right)$. Instead of the critical points $\tilde{x}(t)$, resp. $\hat{x}(t)$ we obtain vector functions $\left(\tilde{y}_{1}(u), \ldots, \tilde{y}_{q}(u)\right)$, resp. $\left(\hat{y}_{1}(u), \ldots, \hat{y}_{q-1}(u), 0\right)$ satisfying:

$$
\begin{aligned}
& \frac{\partial g}{\partial y_{i}}\left(\tilde{y}_{1}(u), \ldots, \tilde{y}_{q}(u), u\right) \equiv 0, \quad i=1, \ldots, q, \\
& \frac{\partial g}{\partial y_{i}}\left(\hat{y}_{1}(u), \ldots, \hat{y}_{q-1}(u), 0, u\right) \equiv 0, \quad i=1, \ldots, q-1 .
\end{aligned}
$$

moreover, $\mu_{p}(t)$ (recall (24)) becomes $\mu(u)$, where

$$
\mu(u):=\frac{\partial g}{\partial y_{q}}\left(\hat{y}_{1}(u), \ldots, \hat{y}_{q-1}(u), 0, u\right),
$$

and the corresponding $\gamma$, say $\gamma^{\prime}$, becomes $\left(\mathrm{d} \tilde{y}_{q} / \mathrm{d} u\right)(0)$.

Put $\tilde{G}=D_{y}^{2} g(0)$ and let $\hat{G}$ be the $(q-1) \times(q-1)$ matrix obtained from $\tilde{G}$ by deleting the last column and last row. In view of (A4), (A5) we see that $\tilde{G}$ and $\hat{G}$ are both nonsingular. Furthermore, we put $g_{u, i}=\left(\partial^{2} g / \partial u \partial y_{i}\right)(0), i=1, \ldots, q$, and $g_{i, j}=\left(\partial^{2} g / \partial y_{i} \partial y_{j}\right)(0)$. 
Differentiation of (25), (26) yields:

$$
\begin{aligned}
\frac{\mathrm{d} \mu}{\mathrm{d} u}(0) & =\left[-\left(g_{1, q}, \ldots, g_{q-1, q}\right) \cdot \hat{G}^{-1}, 1\right] \cdot\left(g_{u, 1}, \ldots, g_{u, q}\right)^{\mathrm{T}}, \\
\gamma^{\prime} & =-e_{q}^{\mathrm{T}} \cdot \tilde{G}^{-1} \cdot\left(g_{u, 1}, \ldots, g_{u, q}\right)^{\mathrm{T}}, \quad \text { where } e_{q}=(0, \ldots, 0,1)^{\mathrm{T}} .
\end{aligned}
$$

A short calculation shows:

$$
e_{q}^{\mathrm{T}} \tilde{G}^{-1}=\left[e_{q}^{\mathrm{T}} \tilde{G}^{-1} e_{q}\right]\left[-\left(g_{1, q}, \ldots, g_{q-1, q}\right) \cdot \hat{G}^{-1}, 1\right] .
$$

Now, substitution of (28) in (27) yields the relation:

$$
\gamma^{\prime}=-\frac{\mathrm{d} \mu}{\mathrm{d} u}(0) \cdot\left[e_{q}^{\mathrm{T}} \tilde{G}^{-1} e_{q}\right]
$$

and hence

$$
\operatorname{sign}\left(\gamma^{\prime}\right) \cdot \operatorname{sign}\left(\frac{\mathrm{d} \mu}{\mathrm{d} u}(0)\right)=-\operatorname{sign}\left[e_{q}^{\mathrm{T}} \tilde{G}^{-1} e_{q}\right] .
$$

Let the symbol \# denote 'the number of negative eigenvalues'.

Note that we have:

$$
\operatorname{sign}\left[e_{q}^{\mathrm{T}} \tilde{G}^{-1} e_{q}\right]=+1(-1) \quad \text { iff }\left.\quad \# \tilde{G}^{-1}\right|_{\operatorname{span}\left\{e_{q}\right\}}=0(1) .
$$

The characteristic number $\delta$ becomes

$$
\delta=\# \tilde{G}-\# \hat{G} .
$$

We have to show (cf. (24))

$$
\operatorname{sign}\left(\gamma^{\prime}\right) \cdot \operatorname{sign}\left(\frac{\mathrm{d} \mu}{\mathrm{d} u}(0)\right)=+1 \quad(-1) \quad \text { iff } \quad \delta=1(0) .
$$

In view of (29), (30), (31), it suffices to show the following equality:

$$
\# \tilde{G}-\# \hat{G}=\left.\# \tilde{G}^{-1}\right|_{\text {span }\left\{e_{q}\right\}} \text {. }
$$

The equality (33) is a direct consequence of the following lemma (recall the definition of $\tilde{G}$ and $\hat{G}$ ). Its proof is given in [11], in case $\operatorname{dim} L=n-1$. However, the idea of that proof fits the general case as well, and therefore we delete the proof here.

Lemma 3.2.1. Let $A$ be a nonsingular symmetric $n \times n$ matrix, $L$ a linear subspace of $\mathbb{R}^{n}$ and $L^{\perp}$ its orthogonal complement. Suppose that $\left.A^{-1}\right|_{L^{\perp}}$ is nonsingular. Then we have
a. $\left.A\right|_{L}$ is nonsingular,
b. $\#(A)=\#\left(\left.A\right|_{L}\right)+\#\left(\left.A^{-1}\right|_{L^{1}}\right.$. 


\subsection{Points of Type 3}

A generalized critical point $\bar{z}=(\bar{x}, \bar{t})$ is of Type 3 if the following conditions B1-B4 hold:

B1. $\bar{x}$ is a critical point for $\mathscr{P}(\bar{t})$.

After renumbering we may assume in case $J_{0}(\bar{z}) \neq \emptyset$ that $J_{0}(\bar{z})=\{1, \ldots, p\}$. From B1 and (7) we see that the critical point relation (18) holds.

B2. In (18) we have $\bar{\mu}_{j} \neq 0, j=1, \ldots, p$.

Let the Lagrange function $L$ be defined as in (17) and let the tangent space $T$ be as in (19).

B3. Exactly one eigenvalue of $\left.D_{x}^{2} L(\bar{z})\right|_{T}$ vanishes.

Let $V$ be a matrix with $n$ rows, whose columns form a basis for the tangent space $T$. According to $\mathrm{B} 3$, let $w$ be a nonvanishing vector such that $V^{\mathrm{T}} D_{x}^{2} L(\bar{z}) V w=0$, and put $v=V w$. Put $\Phi=\left(h_{1}, \ldots, h_{m}, g_{1}, \ldots, g_{p}\right)^{\mathrm{T}}$ and define (the symbol ${ }^{\dagger}$ denoting as in (21) the Moore-Penrose inverse):

$$
\begin{aligned}
& \beta_{1}=v^{\mathrm{T}}\left(D_{x}^{3} L \cdot v\right) v-3 v^{\mathrm{T}} D_{x}^{2} L \cdot\left(\left(D_{x}^{\mathrm{T}} \Phi\right)^{\dagger}\right)^{\mathrm{T}}\left(v^{\mathrm{T}} D_{x}^{2} \Phi v\right), \\
& \beta_{2}=D_{t}\left(D_{x} L \cdot v\right)-D_{t}^{\mathrm{T}} \Phi \cdot\left(D_{x}^{\mathrm{T}} \Phi\right)^{\dagger} \cdot D_{x}^{2} L v,
\end{aligned}
$$

where

$$
\begin{aligned}
& v^{\mathrm{T}}\left(D_{x}^{3} L \cdot v\right) v=\sum_{i, j, k=1}^{n}\left(\partial^{3} / \partial x_{i} \partial x_{j} \partial x_{k}\right) L \cdot v_{i} v_{j} v_{k}, \\
& v^{\mathrm{T}} D_{x}^{2} \Phi v=\left(v^{\mathrm{T}} D_{x}^{2} h_{1} v, \ldots, v^{\mathrm{T}} D_{x}^{2} g_{p} v\right)^{\mathrm{T}},
\end{aligned}
$$

all partial derivatives being evaluated at $\bar{z}$. In case that $I=J_{0}(\bar{z})=\emptyset$ we have $T=\mathbb{R}^{n}$ and we delete the entries of $\Phi$ in (34), (35).

Next we define:

$$
\beta=\beta_{1} \cdot \beta_{2} \text {. }
$$

B4. $\beta \neq 0$.

We note that $v$ is determined up to a scalar and hence, $\beta$ is determined up to a positive scalar.

Let $\alpha$ denote the number of negative eigenvalues of $\left.D_{x}^{2} L(\bar{z})\right|_{T}$.

Characteristic numbers: $\alpha, \operatorname{sign}(\beta)$.

Before we proceed with a local analysis of the set $\Sigma$, let us firstly present the easiest example. In fact take the one-dimensional, unconstrained case: $f(x, t)=$ $\frac{1}{3} x^{3}+t x$. The point $\bar{z}$ is now the origin and for the set $\Sigma$ we obtain: $\Sigma=$ $\left\{(x, t) \mid x^{2}+t=0\right\}$. Obviously, $\Sigma$ is a parabola. Note that $\left(\partial^{2} f / \partial x^{2}\right)(0)=0$. This corresponds with condition B3. However, both $\left(\partial^{3} f / \partial x^{3}\right)$ and $\left(\partial^{2} f / \partial t \partial x\right)$ do not vanish at the origin (cf. $\beta_{1}, \beta_{2}$ in (34), (35)). In fact, condition B4 is a generalization of this. 
Now we consider the general case. From condition B1 and Definition 1.2 we see that the set $\left\{D_{x} h_{i}(\bar{z}), D_{x} g_{j}(\bar{z}), i \in I, j \in J_{0}(\bar{z})\right\}$ is linearly independent, and from B2 we learn that all Lagrange parameters $\bar{\mu}_{j}, j \in J_{0}(\bar{z})$ are unequal to zero. From this we conclude that-in some neighbourhood $\hat{O}$ of $\bar{z}$ - the set $\Sigma$ consists entirely of critical points and, moreover, that $J_{0}(z)=J_{0}(\bar{z})$ for all $z \in \Sigma \cap \mathcal{O}$. The latter fact follows from the observation that the set $\left.\left\{D_{x} f, D_{x} h_{i}, i \in I, D_{x} g_{j}, j \in \tilde{J}\right\}\right|_{\bar{z}}$ is linearly independent if $\tilde{J} \subset J_{0}(\bar{z})$ and $\tilde{J} \neq J_{0}(\bar{z})$. But then, we may-locally-consider the inequality constraint functions $g_{j}, j \in J_{0}(\bar{z})$ as equality constraint functions in order to describe the structure of the set $\Sigma$.

For equality-constrained problems, the present type has been studied extensively in [11, 'Type 2']. Therefore, we may restrict ourselves to a citation of those results within this context. In fact, in a neighbourhood of $\bar{z}$, the set $\Sigma$ is a one-dimensional $C^{2}$-manifold. Moreover, the parameter $t$, viewed at as a function on $\Sigma$, has a (nondegenerate) local maximum, resp. local minimum, at $(\bar{x}, \bar{t})$ according to $\operatorname{sign}(\beta)=+1$, resp. $\operatorname{sign}(\beta)=-1$. Consequently, the set $\Sigma$ can be approximated by means of a parabola, in a neighbourhood of $\bar{z}$. The orientation of this parabola as well as the index-relations are depicted in Fig. 3. In view of condition B2, the indices LI, LCI do not change when passing the point $\bar{z}$ along $\Sigma$. Moreover, apart from the degenerate critical point $\bar{z}$, we have $\mathrm{QI}+\mathrm{QCI}=n-|I|-\left|J_{0}(\bar{z})\right|$. Therefore, in Fig. 3 we only mention the change of QI.

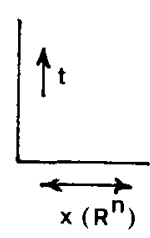

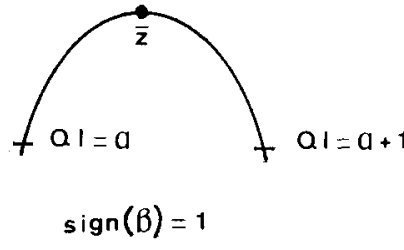

Fig. 3.

\subsection{Points of Type 4}

A generalized critical point $\bar{z}=(\bar{x}, \bar{t})$ is of Type 4 if the following conditions C1-C6 hold:

C1. $|I|+\left|J_{0}(\bar{z})\right|>0, \quad|\cdot|$ denoting the cardinality.

After renumbering we may assume in case $J_{0}(\bar{z}) \neq \emptyset$, that $J_{0}(\bar{z})=\{1, \ldots, p\}$.

C2. dim. $\operatorname{span}\left\{D_{x} h_{i}(\bar{z}), D_{x} g_{j}(\bar{z}), i \in I, j \in J_{0}(\bar{z})\right\}=m+p-1$.

C3. $m+p-1<n$.

From C2 we see that there exist $\lambda_{i}, \mu_{j}, i \in I, j \in J_{0}(\bar{z})$, not all vanishing such that

$$
\sum_{i=1}^{m} \lambda_{i} D_{x} h_{i}(\bar{z})+\sum_{j=1}^{p} \mu_{j} D_{x} g_{j}(\bar{z})=0 .
$$

Note that the numbers $\lambda_{i}, \mu_{j}$ in (37) are unique up to a common multiple.

C4. In case $p \neq 0$, we have $\mu_{j} \neq 0, j=1, \ldots, p$, and we normalize the $\mu_{j}$ 's by setting $\mu_{p}=1$ (normalization). 
We define furthermore

$$
L(z)=\sum_{i=1}^{m} \lambda_{i} h_{i}(z)+\sum_{j=1}^{p} \mu_{j} g_{j}(z),
$$

where $\lambda_{i}, \mu_{j}$ in (38) satisfy (37),

$$
T=\bigcap_{i \in I} \operatorname{Ker} D_{x} h_{i}(\bar{z}) \cap \bigcap_{j \in J_{0}(\bar{z})} \operatorname{Ker} D_{x} g_{j}(\bar{z}) .
$$

Let $W$ be a matrix with $n$ rows, whose columns form a basis for $T$. Define

$$
\begin{aligned}
& A=D_{t} L \cdot W^{\mathrm{T}} \cdot D_{x}^{2} L \cdot W, \\
& w=W^{\mathrm{T}} \cdot D_{x}^{\mathrm{T}} f,
\end{aligned}
$$

all partial derivatives being evaluated at $\bar{z}$.

C5. $A$ is nonsingular.

Finally, define

$$
\alpha=w^{\mathrm{T}} A^{-1} w .
$$

C6. $\alpha \neq 0$.

We remark that $\alpha$ is independent of the choice of the matrix $W$. Let $\beta$ denote the number of positive eigenvalues of $A$. In case $p \neq 0$, let $\gamma$ be the number of negative $\mu_{j}, j \in\{1, \ldots, p-1\}$ and put $\delta=D_{t} L(\bar{z})$. Note, in particular, that $\gamma<p$.

Characteristic numbers: $\operatorname{sign}(\alpha), \beta$.

Characteristic numbers (corresponding to $\mu_{p}=1$ ): $\gamma, \operatorname{sign}(\delta)$.

We proceed with an analysis of the set $\Sigma$. Note that $\bar{x}$ is not a critical point for $\mathscr{P}(\bar{t})$. In fact, from (37) we see that the vectors $D_{x} h_{i}(\bar{z}), D_{x} g_{j}(\bar{z}), i \in I, j \in J_{0}(\bar{z})$, are not linearly independent. In the case $J_{0}(\bar{z})=\emptyset$ we are dealing-locally-only with equality constraints. The equality-constrained case is treated extensively in [11, 'Type 3']. Now we will generalize the corresponding results obtained in [11] for the case that $J_{0}(\bar{z}) \neq \emptyset$. In accordance with the conditions C1-C6 we assume that $J_{0}(\bar{z})=\{1, \ldots, p\}$ and, in addition, that $p \geqslant 1$. In particular, we use the normalization $\mu_{p}=1$ (cf. condition C4).

Conditions $\mathrm{C} 2$ and $\mathrm{C} 4$ imply that for every $q \in J_{0}(\bar{z})$ the following set is linearly independent:

$$
\left\{D_{x} h_{i}(\bar{z}), D_{x} g_{j}(\bar{z}), i \in I, j \in J_{0}(\bar{z}) \backslash\{q\}\right\} .
$$

From condition $\mathrm{C} 5$ and (43) it follows that there exists a neighbourhood $\mathscr{O}$ of $\bar{z}$ such that $\left\{D_{x} h_{i}(z), D_{x} g_{j}(z), i \in I, j \in J_{0}(z)\right\}$ is linearly independent for all $z \in \mathscr{O} \backslash\{\bar{z}\}$. But then, all g.c. points-apart from $\bar{z}$-in some neighbourhood of $\bar{z}$ are critical points. Condition C6 implies in particular that $W^{\mathrm{T}} D_{x}^{\mathrm{T}} f \neq 0$. Consequently, $\bar{z}$ is not a critical point if we delete any $g_{q}$ as a constraint function, where $q \in J_{0}(\bar{z})$ (cf. (43)). In particular we obtain that, in some neighbourhood of $\bar{z}$, at all points of $\Sigma$ the active 
set $J_{0}$ is constant $\left(=J_{0}(\bar{z})\right)$. But then, the local structure of $\Sigma$ around $\bar{z}$ is in accordance with the equality-constrained case as treated in [11], of course including the change of the indices QI, QCI. As a consequence, locally around $\bar{z}$, the set $\Sigma$ is a onedimensional $C^{2}$-manifold, and the parameter $t$, viewed at as a function on $\Sigma$, has a (nondegenerate) local maximum, resp. local minimum, at $(\bar{x}, \bar{t})$ corresponding to $\operatorname{sign}(\alpha)=+1$, resp. $\operatorname{sign}(\alpha)=-1$ (cf. [11], where the characteristic numbers have different names). So, locally around $\bar{z}$, the set $\Sigma$ can be approximated by means of a parabola. It remains to compute the change of the linear indices LI, LCI, in relation with the change of the indices QI, QCI. Let $z$ be a critical point, close to $\bar{z}$, and let $\hat{\mu}_{j}(z)$ be the Lagrange parameter corresponding to the inequality constraint function $g_{j}, j \in J_{0}(\bar{z})$. Then, it is not difficult to see that $\left|\hat{\mu}_{j}(z)\right|$ tends to infinity as $z$ tends to $\bar{z}$. Furthermore, taking (37) and condition C4 into account, a moment of reflection shows that, for $j \neq p$, the sign of $\hat{\mu}_{j}(z)$ equals $\operatorname{sign}\left(\mu_{j}\right) \cdot \operatorname{sign}\left(\hat{\mu}_{p}(z)\right)$, with $\mu_{j}$ as in (37). Therefore, we may reduce our considerations to the case $I=\emptyset$ and $p=\left|J_{0}(\bar{z})\right|=1$.

In the case $I=\emptyset, p=1$, we have the following simplification: $L=g_{1}, T=\mathbb{R}^{n}$ (cf. C2), $W=$ identity matrix (without loss of generality), $w=D_{x}^{\mathrm{T}} f$ and thus, $A=D_{t} g_{1} \cdot D_{x}^{2} g_{1}, \alpha=D_{x} f\left[D_{t} g_{1} \cdot D_{x}^{2} g_{1}\right]^{-1} D_{x}^{\mathrm{T}} f$. In particular, $D_{x} f \neq 0$ and $D_{x}^{2} g_{1}$ is nonsingular (at $z=\bar{z})$.

The critical point equation, with $\eta$ denoting the corresponding Lagrange parameter, becomes

$$
\left.\begin{array}{rl}
D_{x} f-\eta D_{x} g_{1} & =0 \\
g_{1} & =0
\end{array}\right\}, \quad z \neq \bar{z} .
$$

Let the symbol \# again denote 'the number of negative eigenvalues'. At a critical point $z(z \neq \bar{z})$ the quadratic index QI equals $\#\left(D_{x}^{2} f-\left.\eta D_{x}^{2} g_{1}\right|_{\text {Ker } D_{x} g_{1}}\right)$, which, in view of (44), equals

$$
\#\left(D_{x}^{2} f-\left.\eta D_{x}^{2} g_{1}\right|_{\text {Ker } D_{x} f}\right) \text {. }
$$

Note that in (45) the number $|\eta|$ tends to infinity as $z$ tends to $\bar{z}$. Furthermore, $\eta^{-1}$ changes sign at $z=\bar{z}$. Since $D_{x}^{2} g_{1}(\bar{z})$ is nonsingular and $\alpha \neq 0$, it follows from Lemma 3.2.1a that $\left.D_{x}^{2} g_{1}(\bar{z})\right|_{\text {Ker } D_{x} f(\bar{z})}$ is nonsingular. But then, for $z$ close to $\bar{z}$, the number in (45) equals

$$
\#\left(-\left.\eta D_{x}^{2} g_{1}\right|_{\operatorname{Ker} D_{x} f}\right) .
$$

From (46) it follows that the quadratic index QI of a critical point $z, z$ close to $\bar{z}$, corresponds to the following list (the partial derivatives being evaluated at $z=\bar{z}$ ):

$$
\left.\begin{array}{ll}
\text { 1. } & \eta>0, D_{t} g_{1}>0: \mathrm{QI}=\#(-B) \\
\text { 2. } & \eta>0, D_{t} g_{1}<0: \mathrm{QI}=\#(B) \\
\text { 3. } & \eta<0, D_{t} g_{1}>0: \mathrm{QI}=\#(B) \\
\text { 4. } & \eta<0, D_{t} g_{1}<0: \mathrm{QI}=\#(-B)
\end{array}\right\}, \quad B=\left.D_{t} g_{1} \cdot D_{x}^{2} g_{1}\right|_{\operatorname{Ker} D_{x} f} .
$$

So it remains to relate $\#\left( \pm D_{t} g_{1} \cdot D_{x}^{2} g_{1}\right)$ with the number $\#\left( \pm\left. D_{t} g_{1} \cdot D_{x}^{2} g_{1}\right|_{\text {Ker } D_{x} f}\right)$. 
This can be accomplished by means of Lemma 3.2.1b. In particular we have:

$$
\#\left(D_{t} g_{1} \cdot D_{x}^{2} g_{1}\right)=\#\left(\left.D_{t} g_{1} \cdot D_{x}^{2} g_{1}\right|_{\text {Ker } D_{x} f}\right)+\#\left(D_{x} f\left[D_{t} g_{1} \cdot D_{x}^{2} g_{1}\right]^{-1} D_{x}^{\mathrm{T}} f\right) \text {. }
$$

Note that $\#\left(D_{x} f\left[D_{t} g_{1} \cdot D_{x}^{2} g_{1}\right]^{-1} D_{x}^{\mathrm{T}} f\right)=+1$ (resp. 0) according to $\operatorname{sign}(\alpha)=-1$ (resp. +1).

Now we return to the general case. Then, in (48), $D_{t} g_{1} \cdot D_{x}^{2} g_{1}$ has to be replaced by $D_{i} L \cdot W^{\mathrm{T}} \cdot D_{x}^{2} L \cdot W$, etc. A few calculations show that the index-relations are as depicted in Fig. 4, where the parabolas are local approximations of the set $\Sigma$. If we
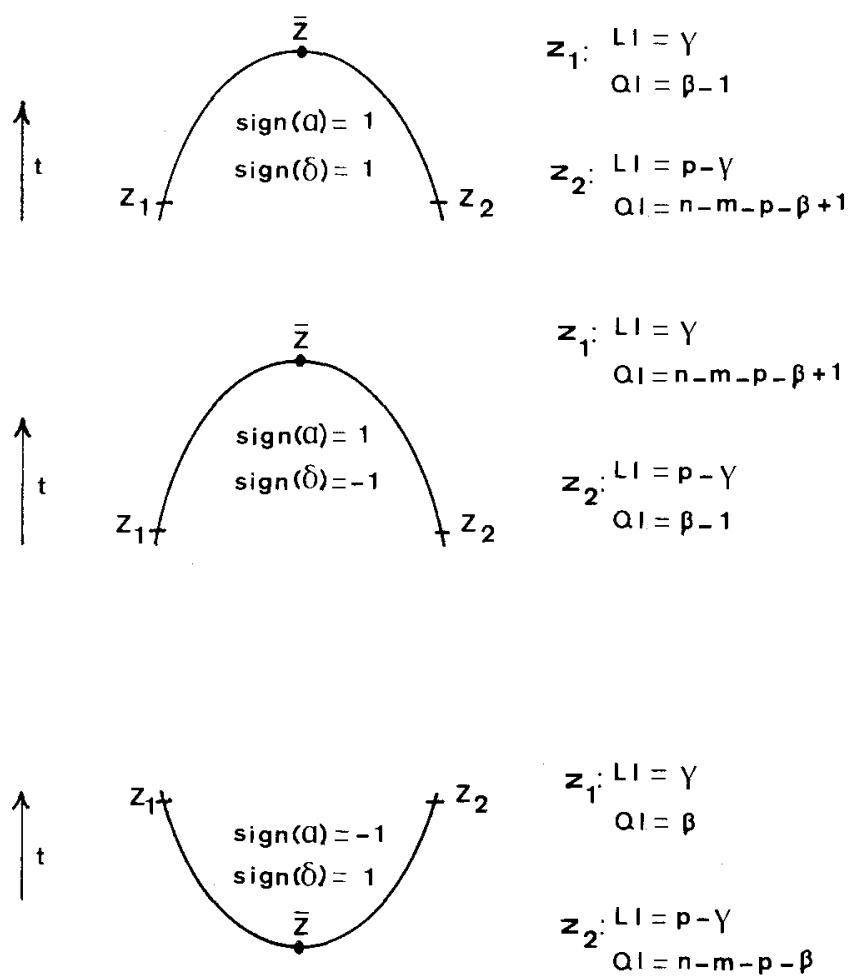

$$
\begin{aligned}
& z_{1}: L I=Y \\
& z_{2}: \begin{array}{l}
L I=p-Y \\
Q I=n-m-p-\beta
\end{array}
\end{aligned}
$$

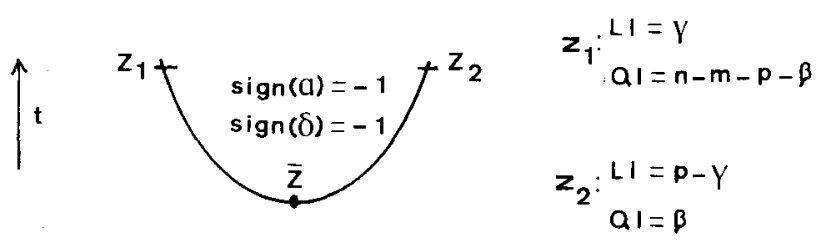

$$
\begin{aligned}
& p=\left|J_{0}(\bar{z})\right|, \quad m=|1|, \text { normalization }: \mu_{p}=1 \\
& L I+L C|=p, \quad a|+Q C \mid=n-m-p
\end{aligned}
$$

Fig. 4. 
would replace $f$ by the function $-f$, this has the following effect on Fig. 4: interchange the index-entries corresponding to $z_{1}, z_{2}$.

\subsection{Points of Type 5}

A generalized critical point $\bar{z}=(\bar{x}, \bar{t})$ is of Type 5 if the following conditions D1-D4 hold:

D1. $|I|+\left|J_{0}(\bar{z})\right|=n+1$.

D2. The set $\left\{D h_{i}(\bar{z}), D g_{j}(\bar{z}), i \in I, j \in J_{0}(\bar{z})\right\}$ is linearly independent (derivatives in $\left.\mathbb{R}^{n+1}\right)$.

Since $m=|I|<n$ throughout the paper, (49) implies that $\left|J_{0}(\bar{z})\right| \geqslant 2$. After renumbering we assume that $J_{0}(\bar{z})=\{1, \ldots, p\}$. From D1, D2, we see that there exist $\lambda_{i}$, $\mu_{j}, i \in I, j \in J_{0}(\bar{z})$, not all vanishing (unique up to a common multiple) such that:

$$
\sum_{i=1}^{m} \lambda_{i} D_{x} h_{i}(\bar{z})+\sum_{j=1}^{p} \mu_{j} D_{x} g_{j}(\bar{z})=0 .
$$

D3. In (50) we have $\mu_{j} \neq 0, j=1, \ldots, p$.

From D1, D2, it follows that there exist unique numbers $\alpha_{i}, \beta_{j}, i \in I, J \in J_{0}(\bar{z})$, such that

$$
D f(\bar{z})=\sum_{i=1}^{m} \alpha_{i} D h_{i}(\bar{z})+\sum_{j=1}^{p} \beta_{j} D g_{j}(\bar{z}) .
$$

Put

$$
\Delta_{i j}=\beta_{i}-\beta_{j} \cdot \mu_{i} / \mu_{j}, \quad i, j=1, \ldots, p,
$$

and let $\Delta$ be the $p \times p$ matrix with $\Delta_{i j}$ as its $(i, j)$ th element.

D4. All off-diagonal elements of $\Delta$ are unequal to zero.

Put

$$
L(z)=\sum_{i=1}^{m} \lambda_{i} h_{i}(z)+\sum_{j=1}^{p} \mu_{j} g_{j}(z),
$$

where $\lambda_{i}, \mu_{j}$ satisfy $(50)$. From D2 we see that $D_{t} L(\bar{z}) \neq 0$. We define:

$$
\gamma_{j}=\operatorname{sign}\left(\mu_{j} \cdot D_{t} L(\bar{z})\right), \quad j=1, \ldots, p .
$$

By $\delta_{j}$ we denote the number of negative entries in the $j$ th column of $\Delta, j=1, \ldots, p$.

Characteristic numbers: $\gamma_{j}, \delta_{j}, j=1, \ldots, p$.

We proceed with an analysis of the set $\Sigma$. From conditions D1-D3 it follows that for every $q \in\{1, \ldots, p\}$ the following set is linearly independent:

$$
\left\{D_{x} h_{i}(\bar{z}), D_{x} g_{j}(\bar{z}), i \in I, j \in J_{0}(\bar{z}) \backslash\{q\}\right\} .
$$

Next, conditions D1, D2 and the linear independence of (55) for all $q \in J_{0}(\bar{z})=$ $\{1, \ldots, p\}$ imply that there exists a neighbourhood $\mathcal{O}$ of $\bar{z}$ such that $\left\{D_{x} h_{i}(z), D_{x} g_{j}(z), i \in I, j \in J_{0}(z)\right\}$ is linearly independent for all $z \in \mathscr{O} \backslash\{\bar{z}\}$. But then, 
apart from $\bar{z}$, all generalized critical points in some neighbourhood of $\bar{z}$ are critical points. A combination of (50) and (51) implies at $z=\bar{z}$;

$$
D_{x} f=\sum_{i=1}^{m}\left(\alpha_{i}-\beta_{q} \cdot \frac{\lambda_{i}}{\mu_{q}}\right) D_{x} h_{i}+\sum_{j=1}^{p}\left(\beta_{j}-\beta_{q} \frac{\mu_{j}}{\mu_{q}}\right) D_{x} g_{j}, \quad q=1, \ldots, p .
$$

Consequently, for $q=1, \ldots, p$, we obtain that $\bar{z}$ is a nondegenerate critical point if we delete $g_{q}$ as a constraint (combine (56) and condition D4). We put

$$
\begin{aligned}
& M_{q}=\left\{z \mid h_{i}(z)=0, g_{j}(z)=0, i \in I, j \in J_{0}(\bar{z}) \backslash\{q\}\right\}, \quad q=1, \ldots, p, \\
& M_{q}^{+}=\left\{z \in M_{q} \mid g_{q}(z) \geqslant 0\right\} .
\end{aligned}
$$

From conditions D1, D2, and the fact that $h_{i}, g_{j}$ are $C^{3}$-functions, it follows that, locally around $\bar{z}$, the set $M_{q}$ is a one-dimensional $C^{3}$-manifold, $q=1, \ldots, p$. Furthermore, locally around $\bar{z}$, the set $\Sigma$ is equal to the union $\bigcup_{q=1}^{p} M_{q}^{+}$. A moment of reflection shows that there exists a local $C^{3}$-coordinate transformation of a neighbourhood $\mathscr{V}$ of $\bar{z}$ which maps the set $\Sigma \cap \mathscr{V}$ to the union of the coordinate-axes of the orthant $\mathbb{H}^{p}=\left\{y \in \mathbb{R}^{p} \mid y_{i} \geqslant 0, i=1, \ldots, p\right\}$. The indices (LI, LCI, QI, QCI) along $M_{q}^{+} \backslash\{\bar{z}\}$ are equal to $\left(\delta_{q}, p-1-\delta_{q}, 0,0\right)$, where $\delta_{q}$ is a characteristic number as introduced above (combine the definition of $\delta_{q}$ with (52) and (56)).

It is easily seen that the $t$-component of the tangent space of $M_{q}$ at $\bar{z}$ is unequal to zero, $q=1, \ldots, p$. Furthermore, a short calculation shows that, as $t$ increases and passes the value $\bar{t}$, the set $M_{q}^{+}$emanates from $(\bar{x}, \bar{t})$, resp. ends at $(\bar{x}, \bar{t})$ according to $\gamma_{q}=+1$, resp. $\gamma_{q}=-1$, where $\gamma_{q}$ is one of the characteristic numbers (cf. (54)).

\section{The Kuhn-Tucker subset}

In this section we will look more closely to the subset of $\Sigma$ consisting of Kuhn-Tucker points. This set has been studied extensively in the important paper [12] under the additional assumption of the so-called Mangasarian-Fromowitz Constraint Qualification (shortly: MFCQ). However, the MFCQ is not a generic condition in optimization problems depending on parameters. With the aid of the foregoing analysis we are able to describe generically the Kuhn-Tucker subset (without explicitly appealing to the MFCQ) and we will discuss those situations where the MFCQ fails to hold.

We will adopt the notations used in the foregoing sections. In particular, recall that $\mathscr{F}$ denotes the set of generic one-parametric programs (cf. Definition 2.1 and Theorem 2.1) and note that $\Sigma^{1}$ stands for that subset of $\Sigma$, consisting entirely of nondegenerate critical points.

Definition 4.1. The subset $\Sigma_{K T}$ (the 'Kuhn-Tucker subset') of $\Sigma$ is defined to be the closure of the set $\left\{z \in \Sigma^{1} \mid \mathrm{LI}=0\right.$ at $\left.z\right\}$. 
Definition 4.2. Let $\bar{z}=(\bar{x}, \bar{t})$ and let $\bar{x}$ be a feasible point for $\mathscr{P}(\bar{t})$, i.e. $\bar{x} \in M(\bar{t})$. The Mangasarian-Fromowitz Constraint Qualification (MFCQ) is said to be satisfied at $\bar{z}$ if both of the following conditions hold:

a. $\left\{D_{x} h_{i}(\bar{z}), i \in I\right\}$ is a linearly independent set,

b. There exists a vector $\xi \in \mathbb{R}^{n}$ such that

$$
D_{x} h_{i}(\bar{z}) \xi=0, \quad i \in I, \quad D_{x} g_{j}(\bar{z}) \xi>0, \quad j \in J_{0}(\bar{z}) .
$$

Lemma 4.1. Let $\left(f, h_{1}, \ldots, h_{m}, g_{1}, \ldots, g_{s}\right) \in \mathscr{F}$ and $\bar{z} \in \Sigma_{K T}$. Then, at $\bar{z}$, the MFCQ is not satisfied iff

either: $\bar{z}$ is of Type 4, or: $\bar{z}$ is of Type 5, and all $\mu_{j}$ in (50) have the same sign.

Proof. If $\bar{z}$ is of Type 1,2 or 3 , then the set $\left\{D_{x} h_{i}(\bar{z}), D_{x} g_{j}(\bar{z}), i \in I, j \in J_{0}(\bar{z})\right\}$ is linearly independent, and this obviously implies the validity of the MFCQ. Next, suppose that $\bar{z}$ is of Type 4 . If $J_{0}(\bar{z})=\emptyset$, then condition C2 implies that $\left\{D_{x} h_{i}(\bar{z}), i \in I\right\}$ is a linearly dependent set and hence the MFCQ is not satisfied in view of condition $a$ in Definition 4.2. Now, let $J_{0}(\bar{z}) \neq \emptyset$. Since $\bar{z} \in \Sigma_{K T}$ it follows from Fig. 4 that $\gamma=0$ (recall: $\gamma<p$ ). Hence, all $\mu_{j}$ in (37) have the same sign (unequal zero, by taking $\mathrm{C} 4$ into account). But then it is easily seen that a solution $\xi$ of (59) contradicts (37). So, also in this case the MFCQ is not satisfied. Next, let $\bar{z}$ be of Type 5. Since $J_{0}(\bar{z}) \neq \emptyset$ in this case, it follows from condition D3 that the linear independence condition $a$ in Definition 4.2 is satisfied. From the well-known alternative theorems of Farkas' type (e.g. [6]) and condition D3 it follows that (59) is not solvable iff in (50) all $\mu_{j}$ have the same sign. This proves the lemma.

Theorem 4.1. Let $\left(f, h_{1}, \ldots, h_{m}, g_{1}, \ldots, g_{s}\right) \in \mathscr{F}$. Then $\Sigma_{K T}$ is a one-dimensional (piecewise $C^{2}$-) manifold with boundary. In particular, $\bar{z} \in \Sigma_{K T}$ is a boundary point iff at $\bar{z}$ we have: $J_{0}(\bar{z}) \neq \emptyset$ and the MFCQ fails to hold.

Proof. It suffices to consider the structure of $\Sigma_{K T}$ in a neighbourhood of each of the points of Type 1-5. Locally around a point of Type 1 , the set $\Sigma_{K T}$ is a $C^{2}$-manifold (cf. Section 3.1). If $\bar{z}$ is of Type 2, it follows from Fig. 2 that, locally, the structure of $\Sigma_{K T}$ is one of those depicted in Fig. 5. Now, let $\bar{z}$ be of Type 3. Then, the local structure of $\Sigma_{K T}$ coincides with Fig. 3. If $\bar{z}$ is of Type 4, then, in view of Fig. 4,
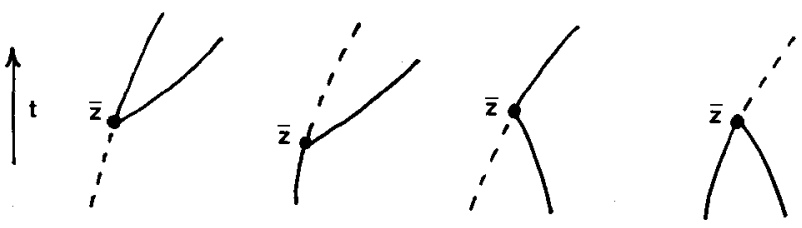

$: \Sigma_{K T}$

Fig. 5. 


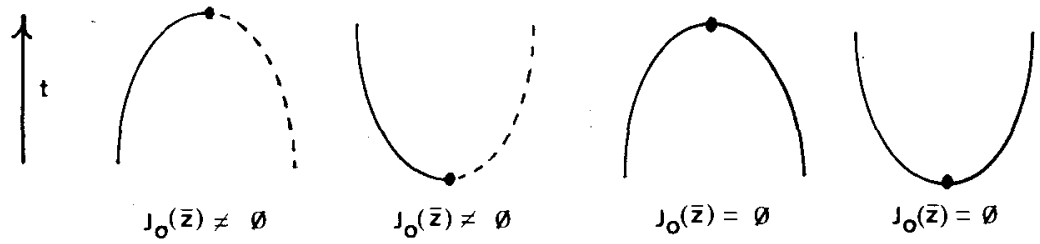

Fig. 6.

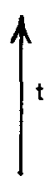

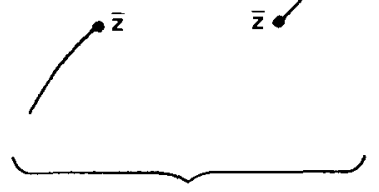

MFCQ fails to hold

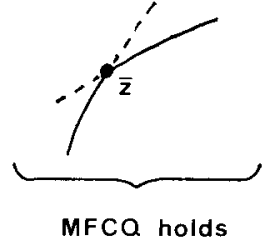

Fig. 7.

the set $\Sigma_{K T}$ has a local structure as depicted in Fig. 6 . Note: $\bar{z}$ is a boundary point of $\Sigma_{K T}$ if $J_{0}(\bar{z}) \neq \emptyset$, and recall Lemma 4.1 .

Finally, let $\bar{z}$ be of Type 5. A short calculation shows that the numbers $\Delta_{i j}$ in (52) satisfy the following relation:

$$
\Delta_{i j}=-\frac{\mu_{i}}{\mu_{j}} \Delta_{j i}, \quad i, j=1, \ldots, p
$$

In case that all $\mu_{i}, i=1, \ldots, p$, have the same sign (and thus, in view of Lemma 4.1, the MFCQ is not satisfied), (60) implies: $\operatorname{sign}\left(\Delta_{i j}\right)=-\operatorname{sign}\left(\Delta_{j i}\right)$. But then, if for some $q \in\{1, \ldots, p\}$ we have $\delta_{q}=0$, it follows that $\delta_{j}>0$ for all $j \in\{1, \ldots, p\} \backslash\{q\}$. Hence, $\bar{z}$ is a boundary point of $\Sigma_{K T}$. Now suppose that $\mu_{j}, j=1, \ldots, p$, do not all have the same sign. Then, the MFCQ is satisfied. From (60) it is easily seen that we have for at most one of the $M_{q}^{+} \backslash\{\bar{z}\}$ (cf. (58)), emanating from $\bar{z}$ (resp. ending at $\bar{z}$ ) as $t$ increases and passes the value $\bar{t}$, that $\mathrm{LI}=0$. A moment of reflection shows that in case $\mathrm{LI}=0$ on $M_{q}^{+} \backslash\{\bar{z}\}, M_{q}^{+}$ending at $\bar{z}$, there exists exactly one $r \in$ $\{1, \ldots, p\} \backslash\{q\}$ such that $M_{r}^{+}$emanates from $\bar{z}$ and $\mathbf{L I}=0$ on $M_{r}^{+} \backslash\{\bar{z}\}$. Altogether, the local structure of $\Sigma_{K T}$ around $\bar{z}$ has one of the forms as depicted in Fig. 7. This completes the proof of our theorem.

\section{Acknowledgement}

We are indebted to the anonymous referees for their rigorous criticism which improved the readability of the original manuscript. 


\section{References}

[1] E.L. Allgower and K. Georg, "Predictor-corrector and simplicial methods for approximating fixed points and zero points of nonlinear mappings", in: A. Bachem, M. Grötschel and B. Korte, eds., Mathematical programming, the state of the art (Springer-Verlag, Berlin, 1983) pp. 15-56.

[2] V.I. Arnol'd, A.N. Varchenko and S.M. Gusein-Zade, Singularities of differentiable maps (Nayka, Moscow, 1982) (in Russian).

[3] A.V. Fiacco, Introduction to sensitivity and stability analysis in nonlinear programming (Academic Press, New York, 1983).

[4] A.V. Fiacco, ed., "Sensitivity, stability and parametric analysis", Mathematical Programming Study 21 (1984).

[5] J. Guddat and K. Wendler, "On dialogue-algorithms for linear and nonlinear vector optimization from the point of view of parametric programming", in: M. Grauer and A.P. Wierzbicki, eds., Interactive decision analysis proceedings, Laxenburg, Austria 1983, Lecture Notes in Economics and Mathematical Systems 229 (Springer-Verlag, Berlin, 1984).

[6] R. Hettich and P. Zencke, Numerische Methoden der Approximation und semi-infiniten Optimierung (Teubner Studienbücher, Stuttgart, 1982).

[7] M.W. Hirsch, Differential topology (Springer-Verlag, Berlin, 1976).

[8] H.Th. Jongen, P. Jonker and F. Twilt, "On deformation in optimization", Methods of Operations Research 37 (1980) 171-184.

[9] H.Th. Jongen, P. Jonker and F. Twilt, "On one-parameter families of sets defined by (in)equality constraints", Nieuw Archief voor Wiskunde (3) 30 (1982) 307-322.

[10] H.Th. Jongen, P. Jonker and F. Twilt, Nonlinear optimization in $\mathbb{R}^{n}, I$. Morse theory, Chebyshev approximation, Methoden und Verfahren der mathematischen Physik 29 (Peter Lang Verlag, Frankfurt a.M., 1983).

[11] H.Th. Jongen, P. Jonker and F. Twilt, "One-parameter families of optimization problems: equality constraints", Journal of Optimization Theory and Applications 48 (1986) 141-161.

[12] M. Kojima and R. Hirabayashi, "Continuous deformation of nonlinear programs", Mathematical Programming Study 21 (1984) 150-198.

[13] M. Marcus and H. Minc, A survey of matrix theory and matrix inequalities (Allyn and Bacon Inc., Boston, 1964).

[14] J. Milnor, Morse theory, Annals of Mathematics Studies 51 (Princeton University Press, Princeton, NJ, 1963).

[15] D. Siersma, "Singularities of functions on boundaries, corners etc.", The Quarterly Journal of Mathematics, Oxford Series (2) 32 (1981) 119-127.

[16] J.E. Spingarn, "On optimality conditions for structured families of nonlinear programming problems", Mathematical Programming 22 (1982) 82-92. 